\title{
Oscillation of Certain Partial Difference Equations
}

\author{
SUNG KYU CHOI ${ }^{\mathrm{a}, *}$ and BING GEN ZHANG ${ }^{\mathrm{b}, \dagger}$ \\ a Department of Mathematics, Chungnam National University, Taejon, 305-764, Korea; \\ ${ }^{\mathrm{b}}$ Department of Applied Mathematics, Ocean University of Qingdao, Qingdao 266003, China
}

(Received 20 May 1998)

We first obtain sufficiency conditions for the oscillation of all solutions of linear partial difference equation

$$
a A_{m+1, n+1}+b A_{m+1, n}+c A_{m, n+1}-d A_{m, n}+P_{m, n} A_{m-k, n-l}=0 .
$$

Next, we establish a linearized oscillation result for the nonlinear partial difference equation

$$
A_{m+1, n+1}+A_{m+1, n}+A_{m, n+1}-A_{m, n}+P_{m, n} f\left(A_{m-k, n-l}\right)=0 .
$$

Keywords: Linear and nonlinear partial difference equations, Oscillation

AMS Subject Classification: 39A10

\section{INTRODUCTION}

Partial difference equations have been posed from various practical problems $[3,8]$ and in the approximation of solutions of partial difference equations by finite difference methods $[1,2,8]$. Recently, the qualitative analysis of partial difference equations has received much attention, see $[4-7,9,10,12]$.
In this paper, we first consider the linear partial difference equation

$$
\begin{array}{r}
a A_{m+1, n+1}+b A_{m+1, n}+c A_{m, n+1} \\
-d A_{m, n}+P_{m, n} A_{m-k, n-l}=0,
\end{array}
$$

where $P_{m, n}>0$ on $N_{0}^{2}, k, l \in N_{0}, N_{i}=\{i, i+1, \ldots\}$ and $i$ is an integer. Equation (1.1) can be regarded

\footnotetext{
* Corresponding author. Supported by Ministry of Education, Korea, BSRI 97-1428. E-mail: skchoi(a)math.chungnam.ac.kr.

${ }^{\dagger}$ Supported by NNSF of China. E-mail: bgzhang $(a)$ lib.ouqd.edu.cn.
} 
as a discrete analogue of the delay partial difference equation

$$
\begin{aligned}
& a \frac{\partial^{2} u}{\partial y \partial x}+a_{1} \frac{\partial u}{\partial x}+a_{2} \frac{\partial u}{\partial y} \\
& \quad+P(x, y) u(x-\sigma, y-\tau)=0 .
\end{aligned}
$$

Next, we consider the nonlinear partial difference equation

$$
\begin{aligned}
& A_{m+1, n+1}+A_{m+1, n}+A_{m, n+1} \\
& \quad-A_{m, n}+P_{m, n} f\left(A_{m-k, n-l}\right)=0,
\end{aligned}
$$

where $f \in C(\mathbb{R}, \mathbb{R})$.

The general theory of partial functional differential equations can be seen from $\mathrm{Wu}$ [11].

A double sequence $\left\{A_{m, n}\right\}$ is said to be a solution of (1.1) if it satisfies (1.1) for $m \geq m_{0}, n \geq n_{0}$. A solution $\left\{A_{i, j}\right\}$ of (1.1) is said to be eventually positive if $A_{i, j}>0$ for all large $i$ and $j$, and eventually negative if $A_{i, j}<0$ for all large $i$ and $j$. It is said to be oscillatory if it is neither eventually positive nor eventually negative.

In Section 2, we shall obtain sufficiency conditions for all solutions of (1.1) to be oscillatory. In Section 3, we shall show a linearized oscillation theorem for (1.3), i.e., we shall show that under some assumptions, (1.3) has the same oscillatory character as an associated linear equation.

\section{EQUATION (1.1)}

We assume that $a, b, c, d$ and $P_{m, n}$ are positive in Eq. (1.1). Define a set $E$ by

$$
E=\left\{\lambda>0 \mid d-\lambda P_{m, n}>0, \text { eventually }\right\} .
$$

\section{TheOREM 2.1 Assume that}

(i) $\lim _{m, n \rightarrow \infty} \sup P_{m, n}>0$;

(ii) for $k \geq l \geq 1$, there exist $M, N \in N_{1}$ such that

$$
\begin{aligned}
& \sup _{\lambda \in E, m \geq M, n \geq N} \lambda \prod_{i=1}^{l}\left(d-\lambda P_{m-i, n-i}\right) \\
& \times \prod_{j=1}^{k-l}\left(d-\lambda P_{m-l-j, n-l}\right)<\left(a+\frac{2 b c}{d}\right)^{\prime} b^{k-l}
\end{aligned}
$$

and for $l \geq k$

$$
\begin{aligned}
& \sup _{\lambda \in E, m \geq M, n \geq N} \lambda \prod_{i=1}^{k}\left(d-\lambda P_{m-i, n-i}\right) \\
& \times \prod_{j=1}^{l-k}\left(d-\lambda P_{m-k, n-k-j}\right)<\left(a+\frac{2 b c}{d}\right)^{k} c^{l-k} .
\end{aligned}
$$

Then every solution of (1.1) oscillates.

Proof Suppose, to the contrary, we let $\left\{A_{m, n}\right\}$ be an eventually positive solution. We define a subset $S$ of the positive numbers as follows:

$$
\begin{aligned}
S(A)= & \left\{\lambda>0 \mid a A_{m+1, n+1}+b A_{m+1, n}\right. \\
& \left.+c A_{m, n+1}\left(d-\lambda P_{m, n}\right) A_{m, n} \leq 0, \text { eventually }\right\} .
\end{aligned}
$$

From (1.1), we have

$$
a A_{m+1, n+1}+b A_{m+1, n}+c A_{m, n+1}<d A_{m, n} .
$$

If $k \geq l$, then

$$
A_{m-k, n-l}>\left(\frac{a}{d}\right)^{l} A_{m-k+l, n}>\left(\frac{a}{d}\right)^{l}\left(\frac{b}{d}\right)^{k-l} A_{m, n} .
$$

If $l \geq k$, then

$$
A_{m-k, n-l}>\left(\frac{a}{d}\right)^{k} A_{m, n-l+k}>\left(\frac{a}{d}\right)^{k}\left(\frac{c}{d}\right)^{l-k} A_{m, n} .
$$

Substituting (2.5) and (2.6) into (1.1) we obtain

$$
\begin{aligned}
& a A_{m+1, n+1}+b A_{m+1, n}+c A_{m, n+1} \\
& -d A_{m, n}+\left(\frac{a}{d}\right)^{l}\left(\frac{b}{d}\right)^{k-l} P_{m, n} A_{m, n}<0
\end{aligned}
$$

and

$$
\begin{aligned}
& a A_{m+1, n+1}+b A_{m+1, n}+c A_{m, n+1} \\
& \quad-d A_{m, n}+\left(\frac{a}{d}\right)^{k}\left(\frac{c}{d}\right)^{l-k} P_{m, n} A_{m, n}<0,
\end{aligned}
$$


respectively. Inequalities (2.7) and (2.8) show that $S(A)$ is nonempty. For $\lambda \in S$, we have eventually

$$
d-\lambda P_{m, n}>0
$$

which implies that $S \subset E$. Due to the condition (i), the set $E$ is bounded, and hence $S(A)$ is bounded. Let $\mu \in S$. Then, from (2.4), we have

$$
A_{m+1, n+1} \leq \frac{d}{b} A_{m, n+1} \quad \text { and } \quad A_{m+1, n+1} \leq \frac{d}{c} A_{m+1, n} .
$$

Hence we obtain

$$
\begin{aligned}
& \left(a+\frac{2 b c}{d}\right) A_{m+1, n+1} \\
& \quad \leq a A_{m+1, n+1}+b A_{m+1, n}+c A_{m, n+1} \\
& \quad \leq\left(d-\mu P_{m, n}\right) A_{m, n} .
\end{aligned}
$$

If $k \geq l$, then

$$
A_{m, n} \leq\left(a+\frac{2 b c}{d}\right)^{-l} \prod_{i=1}^{l}\left(d-\mu P_{m-i, n-i}\right) A_{m-l, n-l}
$$

and

$$
\begin{aligned}
A_{m-l, n-l} & \leq \frac{1}{b}\left(d-\mu P_{m-l-1, n-l}\right) A_{m-l-1, n-l} \\
\leq \cdots & \leq\left(\frac{1}{b}\right)^{k-l} \prod_{j=1}^{k-l}\left(d-\mu P_{m-l-j, n-l}\right) A_{m-k, n-l}
\end{aligned}
$$

Hence

$$
\begin{aligned}
A_{m, n} \leq & \left(a+\frac{2 b c}{d}\right)^{-l} b^{l-k} \prod_{i=1}^{l}\left(d-\mu P_{m-i, n-i}\right) \\
& \times \prod_{j=1}^{k-l}\left(d-\mu P_{m-l-j, n-l}\right) A_{m-k, n-l} .
\end{aligned}
$$

Similarly, if $l \geq k$, then

$$
\begin{aligned}
A_{m, n} \leq & \left(a+\frac{2 b c}{d}\right)^{-k} c^{k-l} \prod_{i=1}^{k}\left(d-\mu P_{m-i, n-i}\right) \\
& \times \prod_{j=1}^{l-k}\left(d-\mu P_{m-k, n-k-j}\right) A_{m-k, n-l} .
\end{aligned}
$$

Substituting (2.9) and (2.10) into (1.3) we find respectively

$$
\begin{aligned}
& a A_{m+1, n+1}+b A_{m+1, n}+c A_{m, n+1}-d A_{m, n} \\
& +P_{m, n}\left(a+\frac{2 b c}{d}\right)^{k} c^{l-k}\left(\prod_{i=1}^{k}\left(d-\mu P_{m-i, n-i}\right)\right. \\
& \left.\quad \times \prod_{j=1}^{l-k}\left(d-\mu P_{m-k, n-k-j}\right)\right)^{-1} A_{m, n} \leq 0, \quad \text { for } l \geq k
\end{aligned}
$$

and

$$
\begin{aligned}
& a A_{m+1, n+1}+b A_{m+1, n}+c A_{m, n+1}-d A_{m, n} \\
& \quad+P_{m, n}\left(a+\frac{2 b c}{d}\right)^{l} b^{k-l}\left(\prod_{i=1}^{l}\left(d-\mu P_{m-i, n-i}\right)\right. \\
& \left.\quad \times \prod_{j=1}^{k-l}\left(d-\mu P_{m-l-j, n-l}\right)\right)^{-1} A_{m, n} \leq 0, \quad \text { for } k \geq l .
\end{aligned}
$$

Hence

$$
\begin{aligned}
& a A_{m+1, n+1}+b A_{m+1, n}+c A_{m, n+1} \\
& -\left(d-P_{m, n}\left(a+\frac{2 b c}{d}\right)^{k} c^{l-k}\right. \\
& \times \sup _{m \geq M, n \geq N}\left[\left(\prod_{i=1}^{k}\left(d-\mu P_{m-i, n-i}\right)\right.\right. \\
& \left.\left.\left.\quad \times \prod_{j=1}^{l-k}\left(d-\mu P_{m-k, n-k-j}\right)\right)^{-1}\right]\right) A_{m, n} \leq 0, \quad l \geq k
\end{aligned}
$$

and

$$
\begin{aligned}
& a A_{m+1, n+1}+b A_{m+1, n}+c A_{m, n+1} \\
& -\left(d-P_{m, n}\left(a+\frac{2 b c}{d}\right)^{l} b^{k-l}\right. \\
& \quad \times \sup _{m \geq M, n \geq N}\left[\left(\prod_{i=1}^{l}\left(d-\mu P_{m-i, n-i}\right)\right.\right. \\
& \left.\left.\left.\quad \times \prod_{j=1}^{k-l}\left(d-\mu P_{m-l-j, n-l}\right)\right)^{-1}\right]\right) A_{m, n} \leq 0, \quad k \geq l .
\end{aligned}
$$


From (2.13) and (2.14) we obtain

$$
\begin{gathered}
\left(a+\frac{2 b c}{d}\right)^{k} c^{l-k}\left(\operatorname { s u p } _ { m \geq M , n \geq N } \left[\left(\prod_{i=1}^{k}\left(d-\mu P_{m-i, n-i}\right)\right.\right.\right. \\
\left.\left.\left.\times \prod_{j=1}^{l-k}\left(d-\mu P_{m-k, n-k-j}\right)\right)^{-1}\right]\right) \in S, \quad l>k
\end{gathered}
$$

and

$$
\begin{gathered}
\left(a+\frac{2 b c}{d}\right)^{l} b^{k-l}\left(\operatorname { s u p } _ { m \geq M , n \geq N } \left[\left(\prod_{i=1}^{l}\left(d-\mu P_{m-i, n-i}\right)\right.\right.\right. \\
\left.\left.\left.\times \prod_{j=1}^{k-l}\left(d-\mu P_{m-l-j, n-l}\right)\right)^{-1}\right]\right) \in S, \quad k>l .
\end{gathered}
$$

On the other hand, (2.2) implies that there exits $\beta \in(0,1)$ such that

$$
\begin{aligned}
& \sup _{\lambda \in E, m \geq M, n \geq N} \lambda \prod_{i=1}^{l}\left(d-\lambda P_{m-i, n-i}\right) \prod_{j=1}^{k-l}\left(d-\lambda P_{m-l-j, n-l}\right) \\
& \leq \beta\left(a+\frac{2 b c}{d}\right)^{l} b^{k-l}, \quad k \geq l
\end{aligned}
$$

and (2.3) implies that there exists $\beta \in(0,1)$ such that

$$
\begin{aligned}
& \sup _{\lambda \in E, m \geq M, n \geq N} \lambda \prod_{i=1}^{k}\left(d-\lambda P_{m-i, n-i}\right) \prod_{j=1}^{l-k}\left(d-\lambda P_{m-k, n-k-j}\right) \\
& \quad \leq \beta\left(a+\frac{2 b c}{d}\right)^{k} c^{l-k}, \quad l \geq k
\end{aligned}
$$

Hence, for $k \geq l$,

$$
\begin{aligned}
& \sup _{m \geq M, n \geq N}\left(\prod_{i=1}^{l}\left(d-\mu P_{m-i, n-i}\right) \prod_{j=1}^{k-l}\left(d-\mu P_{m-l-j, n-l}\right)\right) \\
& \leq \frac{\beta}{\mu}\left(a+\frac{2 b c}{d}\right)^{l} b^{k-1}
\end{aligned}
$$

and for $l \geq k$

$$
\begin{aligned}
& \sup _{m \geq M, n \geq N} \prod_{i=1}^{k}\left(d-\mu P_{m-i, n-i}\right) \prod_{j=1}^{l-k}\left(d-\mu P_{m-k, n-k-j}\right) \\
& \leq \frac{\beta}{\mu}\left(a+\frac{2 b c}{d}\right)^{k} c^{l-k}
\end{aligned}
$$

From (2.15) and (2.18) for $l \geq k,(2.16)$ and (2.17) for $k \geq l$, we have that $\mu / \beta \in S$. Repeating the above procedure, we conclude that $\mu(1 / \beta)^{r} \in S, r=$ $1,2, \ldots$, which contradicts the boundedness of $S$. The proof is complete.

From Theorem 2.1, we can derive an explicit oscillation condition.

COROLlary 2.1 In addition to (i) of Theorem 2.1, assume that, for $k \geq l$,

$$
\begin{aligned}
\lim _{m, n \rightarrow \infty} \inf P_{m, n}=P> & d^{k+1}\left(\left(a+\frac{2 b c}{d}\right)^{l} b^{k-l}\right)^{-1} \\
& \times \frac{k^{k}}{(1+k)^{1+k}}
\end{aligned}
$$

and for $l \geq k$,

$$
\begin{aligned}
\lim _{m, n \rightarrow \infty} \inf P_{m, n}= & P>d^{l+1}\left(\left(a+\frac{2 b c}{d}\right)^{k} b^{l-k}\right)^{-1} \\
& \times \frac{l^{l}}{(1+l)^{1+l}}
\end{aligned}
$$

Then the conclusion of Theorem 2.1 holds.

Proof We see that

$$
\max _{d / P>\lambda>0} \lambda(d-\lambda P)^{k}=\frac{d^{k+1} k^{k}}{P(1+k)^{1+k}} .
$$

Hence (2.19) and (2.20) imply that (2.2) and (2.3) hold. By Theorem 2.1, every solution of (1.1) oscillates. The proof is complete.

Remark Obviously, Theorem 2.1 is true for $a=0$. Hence Theorem 2.1 includes Theorem 2.3 in 
[9] as a special case. From (2.4) we have

$$
\begin{aligned}
A_{m, n} & <\frac{d}{b} A_{m-1, n}<\cdots<\left(\frac{d}{b}\right)^{k} A_{m-k, n} \\
& <\left(\frac{d}{b}\right)^{k}\left(\frac{d}{c}\right) A_{m-k, n-1} \\
& <\cdots<\left(\frac{d}{b}\right)^{k}\left(\frac{d}{c}\right)^{l} A_{m-k, n-l} .
\end{aligned}
$$

Let $\mu \in S$. Then

$$
\begin{aligned}
A_{m, n} \leq & \frac{1}{b}\left(d-\mu P_{m-1, n}\right) A_{m-1, n} \\
\leq & \left(\frac{1}{b}\right)^{k} \prod_{i=1}^{k}\left(d-\mu P_{m-i, n}\right) A_{m-k, n} \\
\leq & \left(\frac{1}{b}\right)^{k}\left(\frac{1}{c}\right) \prod_{i=1}^{k}\left(d-\mu P_{m-i, n}\right) \\
& \times\left(d-\mu P_{m-k, n-1}\right) A_{m-k, n-1} \\
\leq & \left(\frac{1}{b}\right)^{k}\left(\frac{1}{c}\right) \prod_{i=1}^{l}\left(d-\mu P_{m-i, n}\right) \\
& \times \prod_{j=1}^{l}\left(d-\mu P_{m-k, n-j}\right) A_{m-k, n-l} .
\end{aligned}
$$

Substituting the above inequality into (1.1) we obtain

$$
\begin{aligned}
& a A_{m+1, n+1}+b A_{m+1, n}+c A_{m, n+1}-d A_{m, n} \\
& +P_{m, n} b^{k} c^{l}\left[\prod_{i=1}^{k}\left(d-\mu P_{m-i, n}\right)\right. \\
& \left.\quad \times \prod_{j=1}^{l}\left(d-\mu P_{m-k, n-j}\right)\right]^{-1} A_{m, n} \leq 0 .
\end{aligned}
$$

Hence

$$
\begin{aligned}
& a A_{m+1, n+1}+b A_{m+1, n}+c A_{m, n+1} \\
& -\left(d-P_{m, n} b^{k} c^{l}\left[\sup _{m \geq M, n \geq N} \prod_{i=1}^{k}\left(d-\mu P_{m-i, n}\right)\right.\right. \\
& \left.\left.\quad \times \prod_{j=1}^{l}\left(d-\mu P_{m-k, n-j}\right)\right]^{-1}\right) A_{m, n} \leq 0,
\end{aligned}
$$

which implies that

$$
\begin{aligned}
b^{k} c^{l}\left[\sup _{m \geq M, n \geq N} \prod_{i=1}^{k}\left(d-\mu P_{m-i, n}\right)\right. \\
\left.\quad \times \prod_{j=1}^{l}\left(d-\mu P_{m-k, n-j}\right)\right]^{-1} \in S .
\end{aligned}
$$

We are ready to state the following proposition.

THEOREM 2.2 In addition to (i) of Theorem 2.1, further assume that (ii)' there exist $M, N \in N_{1}$ such that

$$
\begin{array}{r}
\sup _{\lambda \in E, m \geq M, n \geq N} \lambda \prod_{i=1}^{k}\left(d-\lambda P_{m-i, n}\right) \\
\times \prod_{j=1}^{l}\left(d-\lambda P_{m-k, n-j}\right)<b^{k} c^{l} .
\end{array}
$$

Then every solution of (1.1) oscillates.

Since

$$
\max _{d / q>\lambda>0} \lambda(d-\lambda P)^{k+l}=\frac{d^{k+l+1}(k+l)^{k+l}}{p(1+k+l)^{1+k+l}}
$$

and (2.21), we have the following result.

COROllary 2.2 In addition to (i) of Theorem 2.1, assume that

$$
\lim _{m, n \rightarrow \infty} \inf P_{m, n}=P>\frac{d^{1+k+l}(k+l)^{k+l}}{b^{k} c^{l}(1+k+l)^{1+k+l}} .
$$

Then every solution of $(1.1)$ is oscillatory.

Example 2.1 Consider the partial difference equation

$$
\begin{aligned}
& A_{m+1, n+1}+e A_{m+1, n}+A_{m, n+1} \\
& \quad-A_{m, n}+\frac{1+e}{e^{4}} A_{m-2, n-2}=0 .
\end{aligned}
$$

It is easy to see that (2.23) satisfies the conditions of Corollary 2.1, so every solution of this equation is oscillatory. In fact, $A_{m, n}=(-e)^{m+n}$ is such a solution. 


\section{EQUATION (1.3)}

We consider (1.3) together with the associate linear equation

$A_{m+1, n+1}+A_{m+1, n}+A_{m, n+1}-A_{m, n}+p A_{m-k, n-l}=0$,

where $p>0, k$ and $l$ are positive integers.

For (3.1), the following result is known [12].

LEMMA 3.1 Every solution of (3.1) oscillates if and only if its characteristic equation

$$
\lambda \mu+\lambda+\mu-1+p \lambda^{-k} \mu^{-l}=0
$$

has no positive roots.

LeMmA 3.2 Assume that every solution (3.1) oscillates. Then there exists $\varepsilon_{0} \in(0, p)$ such that for each $\varepsilon \in\left[0, \varepsilon_{0}\right]$ every solution of the equation

$$
\begin{aligned}
& A_{m+1, n+1}+A_{m+1, n}+A_{m, n+1}-A_{m, n} \\
& \quad+(p-\varepsilon) A_{m-k, n-l}=0
\end{aligned}
$$

also oscillates.

Proof By Lemma 3.1, it is sufficient to prove that

$$
\lambda \mu+\lambda+\mu-1+(p-\varepsilon) \lambda^{-k} \mu^{-l}=0
$$

has no positive roots. Obviously, (3.4) has no positive roots in the region $\lambda \mu+\lambda+\mu-1 \geq 0$.

Let

$$
F(\lambda, \mu)=\lambda \mu+\lambda+\mu-1+p \lambda^{-k} \mu^{-l} .
$$

Since (3.2) has no positive roots, so $F(\lambda, \mu)>0$ for $(\lambda, \mu) \in(0, \infty) \times(0, \infty)$. Thus

$$
\min _{\lambda>0, \mu>0} F(\lambda, \mu)=m>0 .
$$

Let

$$
G(s, t)=s t+s+t-1+\frac{1}{2} p s^{-k} t^{-l},
$$

$\varepsilon_{0} \in(0, p / 2)$ and $\varepsilon_{0} \alpha^{-(k+l)} \leq m / 2$, where

$$
0<\alpha<\min \left\{\left(\frac{p}{2}\right)^{1 /(k+l)},\left(\frac{p}{2}\right)^{1 / k},\left(\frac{p}{2}\right)^{1 / l}\right\} .
$$

For $0<\lambda \leq \alpha, 0<\mu \leq \alpha$, we have

$$
\begin{aligned}
\lambda \mu & +\lambda+\mu-1+(p-\varepsilon) \lambda^{-k} \mu^{-l} \\
> & \lambda \mu+\lambda+\mu-1+\frac{p}{2} \lambda^{-k} \mu^{-l} \\
> & -1+\frac{p}{2} \alpha^{-(k+l)}>0 .
\end{aligned}
$$

For $\lambda>\alpha, \mu>\alpha$, we have

$$
\begin{aligned}
\lambda \mu & +\lambda+\mu-1+(p-\varepsilon) \lambda^{-k} \mu^{-l} \\
& \geq F(\lambda, \mu)-\varepsilon_{0} \lambda^{-k} \mu^{-l} \\
& \geq m-\varepsilon_{0} \alpha^{-(k+l)} \geq \frac{m}{2}>0 .
\end{aligned}
$$

For $0<\lambda \geq \alpha, \mu>\alpha, \lambda \mu+\lambda+\mu<1$, we have

$$
\begin{aligned}
\lambda \mu & +\lambda+\mu-1(p-\varepsilon) \lambda^{-k} \mu^{-l} \\
& >\lambda \mu+\lambda+\mu-1+\frac{p}{2} \lambda^{-k} \mu^{-l} \\
& >1-\frac{p}{2} \alpha^{-k}>0 .
\end{aligned}
$$

Similarly, for $\lambda>\alpha, 0<\mu \leq \alpha, \lambda \mu+\lambda+\mu<1$, we have

$$
\lambda \mu+\lambda+\mu-1+(p-\varepsilon) \lambda^{-k} \mu^{-l}>0 .
$$

We have proved that (3.4) has no positive roots.

We are ready to state the following result.

THEOREM 3.1 Assume that

(i) $\lim _{m, n \rightarrow \infty} \inf P_{m, n}=P>0$,

(ii) $f \in C(\mathbb{R}, \mathbb{R}), \quad x f(x)>0$ as $x \neq 0, \lim _{x \rightarrow 0} f(x) /$ $x=1$.

Then every solution of (3.1) oscillates implies that every solution of (1.3) oscillates.

Proof If not, let $A_{m, n}>0, m \geq m_{0}, n \geq n_{0}$ be a solution (1.3). Then $A_{m, n}$ is decreasing in $m$ and $n$, and hence $\lim _{m, n \rightarrow \infty} A_{m, n}=0, \lim _{m \rightarrow \infty} A_{m, n}=0$ and $\lim _{n \rightarrow \infty} A_{m, n}=0$. Let

$$
\bar{P}_{m, n}=P_{m, n} \frac{f\left(A_{m-k, n-l}\right)}{A_{m-k, n-l}} .
$$


Then $\lim _{m, n \rightarrow \infty} \inf \bar{P}_{m, n}=P$. For each $\varepsilon \in\left(0, \epsilon_{0}\right]$, there exist $M$ and $N$ such that $\bar{P}_{m, n}>p-\varepsilon$, for $m \geq M, n \geq N$. Therefore

$$
\begin{aligned}
& A_{m+1, n+1}+A_{m+1, n}+A_{m, n+1}-A_{m, n} \\
& \quad+(p-\varepsilon) A_{m-k, n-l} \leq 0, \quad m \geq M, \quad n \geq N .
\end{aligned}
$$

Summing it in $n$ from $n(\geq N)$ to $\infty$, we have

$$
\begin{gathered}
\sum_{i=n}^{\infty} A_{m+1, i+1}+\sum_{i=n}^{\infty} A_{m+1, i}-A_{m, n} \\
+(p-\varepsilon) \sum_{i=n}^{\infty} A_{m-k, i-l} \leq 0
\end{gathered}
$$

We rewrite the last inequality in the form

$$
\begin{aligned}
& \sum_{i=n}^{\infty} A_{m+1, i+1}+\sum_{i=n+1}^{\infty} A_{m+1, i}+A_{m+1, n}-A_{m, n} \\
& \quad+(p-\varepsilon) \sum_{i=n}^{\infty} A_{m-k, i-l} \leq 0
\end{aligned}
$$

Summing it in $m$ from $m(\geq M)$ to $\infty$, we obtain

$$
\begin{aligned}
& \sum_{j, i=m, n}^{\infty} A_{j+1, i+1}+\sum_{j=m}^{\infty} \sum_{i=n+1}^{\infty} A_{j+1, i} \\
& -A_{m, n}+(p-\varepsilon) \sum_{j, i=m, n}^{\infty} A_{j-k, i-l} \leq 0 .
\end{aligned}
$$

Hence

$$
\begin{aligned}
A_{m, n} \geq & \sum_{j, i=m, n}^{\infty} A_{j+1, i+1}+\sum_{j=m}^{\infty} \sum_{i=n+1}^{\infty} A_{j+1, i} \\
& +(p-\varepsilon) \sum_{j, i=m, n}^{\infty} A_{j-k, i-l} .
\end{aligned}
$$

Define a set of real double sequences

$$
\begin{aligned}
X=\{ & \left\{B_{m, n}\right\} \mid 0 \leq B_{m, n} \leq 1, \\
& m \geq M-k, n \geq N-l\}
\end{aligned}
$$

and an operator $T$ on $X$ by

$$
(T B)_{m, n}=\left\{\begin{array}{c}
\frac{1}{A_{m, n}}\left[2 \sum_{j, i=m+1, n+1}^{\infty} A_{j, i} B_{j, i}\right. \\
\left.+(p-\varepsilon) \sum_{j, i=m, n}^{\infty} A_{j-k, i-l} B_{j-k, i-l}\right] \\
m \geq M, n \geq N, \\
\text { otherwise. }
\end{array}\right.
$$

In view of (3.5), it is easy to see that $T X \subset X$. Define a set of sequences $\left\{B_{m, n}^{(r)}\right\}, r=0,1,2, \ldots$ as follows:

$$
B_{m, n}^{(0)} \equiv 1, \quad B_{m, n}^{(r)}=(T B)_{m, n}^{(r-1)}, \quad r=1,2, \ldots
$$

In view of (3.5), we have

$$
\begin{gathered}
B_{m, n}^{(0)} \geq B_{m, n}^{(1)} \geq \cdots \geq B_{m, n}^{(r)} \geq \cdots, \\
\quad \text { for } m \geq M-k, n \geq N-l .
\end{gathered}
$$

Hence $\lim _{r \rightarrow \infty} B_{m, n}^{(r)}=B_{m, n}$ exists, for $m \geq M-k$, $n \geq N-l$. From (3.6),

$$
B_{m, n}=\left\{\begin{array}{c}
\frac{1}{A_{m, n}}\left[2 \sum_{j, i=m+1, n+1}^{\infty} A_{j, i} B_{j, i}\right. \\
\left.+(p-\varepsilon) \sum_{j, i=m, n}^{\infty} A_{j-k, i-l} B_{j-k, i-l}\right] \\
m \geq M, n \geq N \\
\quad \text { otherwise. }
\end{array}\right.
$$

Clearly, $B_{m, n}>0$ for $m \geq M-k, n \geq N-l$. Let $x_{m, n}=A_{m, n} B_{m, n}$. Then $x_{m, n}>0$ for $m \geq M-k$, $n \geq N-l$ and

$$
x_{m, n}=2 \sum_{j=m+1}^{\infty} \sum_{i=n+1}^{\infty} x_{j, i}+(p-\varepsilon) \sum_{j=m}^{\infty} \sum_{i=n}^{\infty} x_{j-k, i-l} .
$$

Hence

$$
x_{m+1, n}-x_{m, n}=-2 \sum_{i=n+1}^{\infty} x_{m+1, i}-(p-\varepsilon) \sum_{i=n}^{\infty} x_{m-k, i-l}
$$

or

$x_{m, n}=\sum_{i=n+1}^{\infty} x_{m+1, i}+\sum_{i=n}^{\infty} x_{m+1, i}+(p-\varepsilon) \sum_{i=n}^{\infty} x_{m-k, i-l}$. 
Then we have

$$
\begin{aligned}
x_{m, n+1}-x_{m, n}= & -x_{m+1, n+1}-x_{m+1, n} \\
& -(p-\varepsilon) x_{m-k, n-l}=0,
\end{aligned}
$$

which implies that (3.3) has a positive solution $\left\{x_{m, n}\right\}$. By Lemma 3.2, (3.1) has a positive solution, which is a contradiction. The proof is complete.

\section{THEOREM 3.2 Assume that}

(i) $0 \leq P_{m, n} \leq P$,

(ii) there exists $h>0$ such that $f(x)$ is nondecresing and $0 \leq f(x) / x \leq 1$, for $0<|x|<h$.

If (3.1) has a positive solution, then (1.3) also has a positive solution.

Proof If (3.1) has a positive solution, by Lemma 3.1 ,

$$
\lambda \mu+\lambda+\mu-1+p \lambda^{-k} \mu^{-l}=0
$$

has a positive $\operatorname{root}(\lambda, \mu)$ with $0<\lambda<1,0<\mu<1$ and that $\left\{\lambda^{m} \mu^{n}\right\}$ is a positive solution of (3.1). Choose $a>0$ such that

$$
A_{m, n}=a \lambda^{m} \mu^{n}<h, \quad \text { for } m \geq-k, n-l .
$$

$\left\{A_{m, n}\right\}$ is a positive solution of (3.1) and satisfies $f\left(A_{m, n}\right) \leq A_{m, n}$ by condition (ii). Similar to Theorem 3.1, summing (3.1) we get

$$
\begin{gathered}
A_{m, n}=2 \sum_{j=m+1}^{\infty} \sum_{i=n+1}^{\infty} A_{j, i}+p \sum_{j=m}^{\infty} \sum_{i=n}^{\infty} A_{j-k, i-l}, \\
m \geq 0, \quad n \geq 0 .
\end{gathered}
$$

Hence

$$
\begin{aligned}
A_{m, n} \geq & 2 \sum_{j=m+1}^{\infty} \sum_{i=n+1}^{\infty} A_{j, i} \\
& +\sum_{j=m}^{\infty} \sum_{i=n}^{\infty} P_{j, i} f\left(A_{j-k, i-l}\right) .
\end{aligned}
$$

Define

$$
X=\left\{\left\{B_{m, n}\right\} \mid 0 \leq B_{m, n} \leq 1, m \geq-k, n \geq-l\right\}
$$

and an operator $T$ on $X$ by

$$
\left(T B_{m, n}\right)=\left\{\begin{array}{c}
\frac{1}{A_{m, n}}\left[2 \sum_{j=m+1}^{\infty} \sum_{i=n+1}^{\infty} A_{j, i} B_{j, i}\right. \\
\left.+\sum_{j=m}^{\infty} \sum_{i=n}^{\infty} P_{j, i} f\left(A_{j-k, i-l} B_{j-k, i-l}\right)\right] \\
m \geq 0, n \geq 0 \\
\quad \text { otherwise. }
\end{array}\right.
$$

In view of (3.7), $T X \subset X$. Similar to Theorem 3.1, we can prove that there exists $\left\{B_{m, n}\right\} \in X$ such that $(T B)_{m, n}=B_{m, n}$ for $m \geq 0, n \geq 0$. Let $x_{m, n}=$ $A_{m, n} B_{m, n}>0, m \geq 0, n \geq 0$. Then

$$
x_{m, n}=2 \sum_{j=m+1}^{\infty} \sum_{i=n+1}^{\infty} x_{j, i}+\sum_{j=m}^{\infty} \sum_{i=n}^{\infty} P_{j, i} f\left(x_{j-k, i-l}\right) .
$$

Similar to the proof of Theorem 3.1, (3.8) implies that $\left\{x_{m, n}\right\}$ is a positive solution of (1.3). The proof is complete.

From Theorems 3.1 and 3.2 we obtain

Corollary 3.1 Assume that $P_{m, n} \equiv P>0$, (ii) of Theorem 3.1 and (ii) of Theorem 3.2 hold. Then every solution of (1.3) oscillates if and only if every solution of (3.1) oscillates.

Consider (1.3) together with the equation

$$
\begin{aligned}
& A_{m+1, n+1}+A_{m+1, n}+A_{m, n+1} \\
& \quad-A_{m, n}+q_{m, n} f\left(A_{m-k, n-l}\right)=0,
\end{aligned}
$$

where $f \in C(\mathbb{R}, \mathbb{R}), x f(x)>0$ as $x \neq 0$.

We have the comparison theorem as follows:

THEOREM 3.3 Assume that $p_{m, n} \geq q_{m, n}>0$ for all large $m, n$ and that every solution of (3.9) oscillates. Then every solution of (1.3) oscillates.

Proof Suppose to the contrary, $\left\{A_{m, n}\right\}$ is a positive solution, of (1.3). Summing (1.3), we 
obtain

$$
\begin{aligned}
A_{m, n} & =2 \sum_{j=m+1}^{\infty} \sum_{i=n+1}^{\infty} A_{j, i}+\sum_{j=m}^{\infty} \sum_{i=n}^{\infty} p_{j, i} f\left(A_{j-k, i-l}\right) \\
& \geq 2 \sum_{j=m+1}^{\infty} \sum_{i=n+1}^{\infty} A_{j, i}+\sum_{j=m}^{\infty} \sum_{i=n}^{\infty} q_{j, i} f\left(A_{j-k, i-l}\right),
\end{aligned}
$$

from which and using the method in the proof of Theorem 3.2, we obtain a positive solution of (3.9). This contradiction proves the theorem.

\section{References}

[1] R. Courant, K. Friedrichs and H. Lewy, On partial difference equations of mathematical physics, IBMJ, 11 (1967), 215-234.

[2] R.P. Agarwal, Difference Equation and Inequalities, Marcel Dekker, New York (1992).

[3] X.P. Li, Partial difference equations used in the study of molecular orbits (in Chinese), Acta Chimica SINICA, 40 (1982), 688-698.
[4] B.G. Zhang, S.T. Liu and S.S. Cheng, Oscillation of a class of delay partial difference equations, J. Difference Eq. Appl., 1 (1995), 215-226.

[5] B.G. Zhang and S.T. Liu, Oscillation of partial difference equations, Pan American Math. J., 5 (1995), 61-70.

[6] S.C. Fu and L.Y. Tsai, Oscillation in nonlinear difference equations, Advance in Difference Equations II, Computers Math. Appl. (to appear)

[7] B.G. Zhang and S.T. Liu, Oscillation of partial difference equations with variable coefficients, Advance in Difference Equations II, Computers Math. Appl. (to appear).

[8] R.E. Mickens, Difference Equations, Theory and Applications, Van Nostrand Reinhold, New York (1990).

[9] B.G. Zhang and S.T. Liu, On the oscillation of two partial difference equations, J. Math. Anal. Appl., 206 (1997), $480-492$.

[10] B.G. Zhang and S.T. Liu, Necessary and sufficient conditions for oscillations of partial difference equations, Nonlinear Studies, 3 (1996), 187-192.

[11] Jianhong Wu, Theory and Applications of Partial Functional Differential Equations, Springer-Verlag, New York (1996).

[12] B.G. Zhang and S.T. Liu, Necessary and sufficient conditions for oscillations of linear delay partial difference equations, Discrete Dynamics in Nature and Society, 1 (1998), 265-268. 


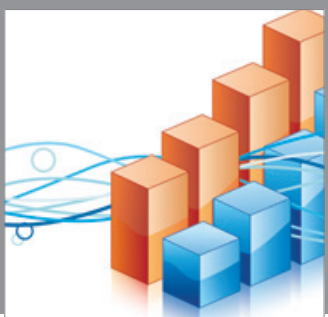

Advances in

Operations Research

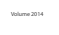

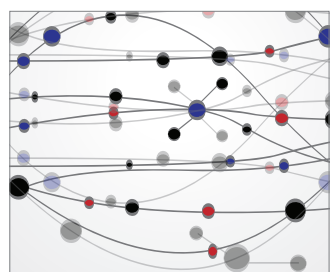

\section{The Scientific} World Journal
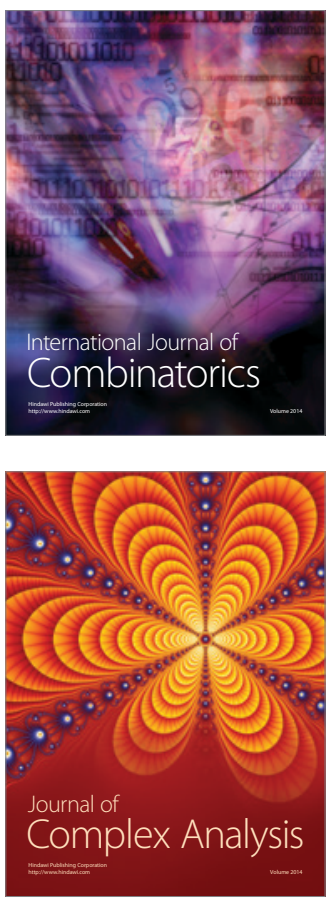

International Journal of

Mathematics and

Mathematical

Sciences
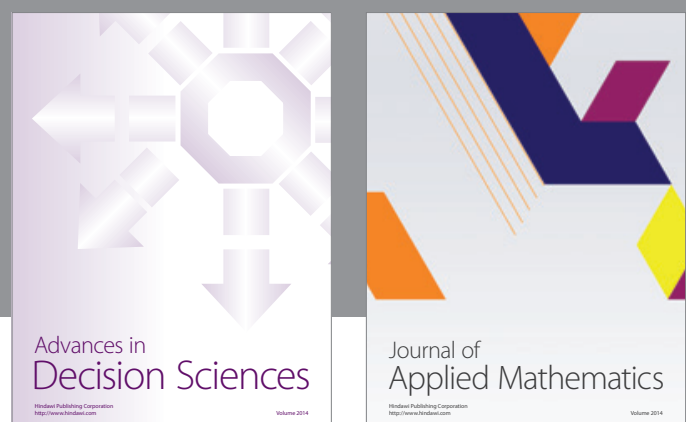

Journal of

Applied Mathematics
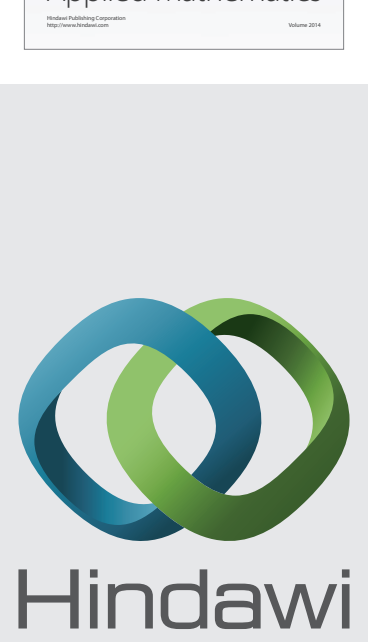

Submit your manuscripts at http://www.hindawi.com
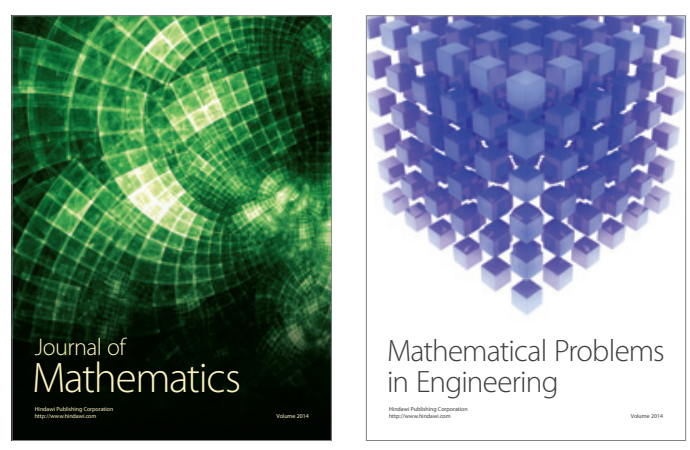

Mathematical Problems in Engineering
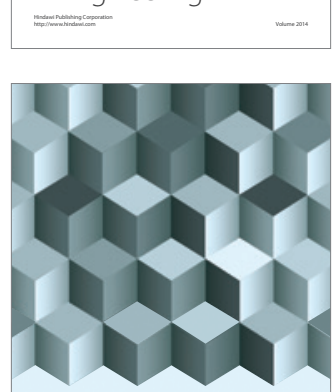

Journal of

Function Spaces
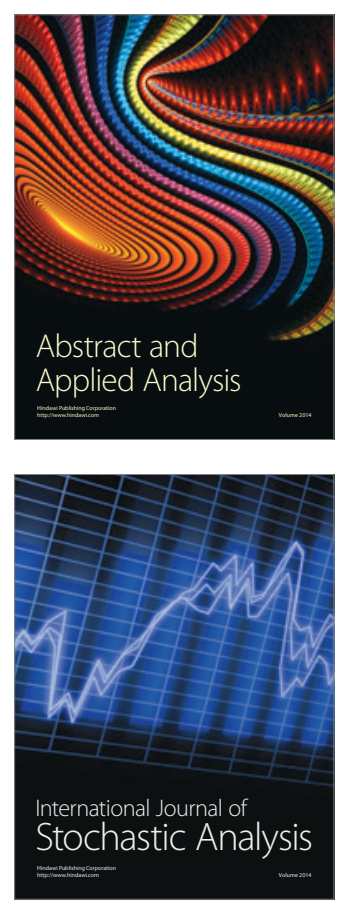

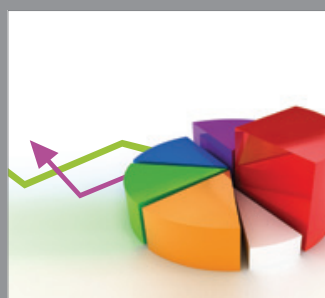

ournal of

Probability and Statistics

Promensencen
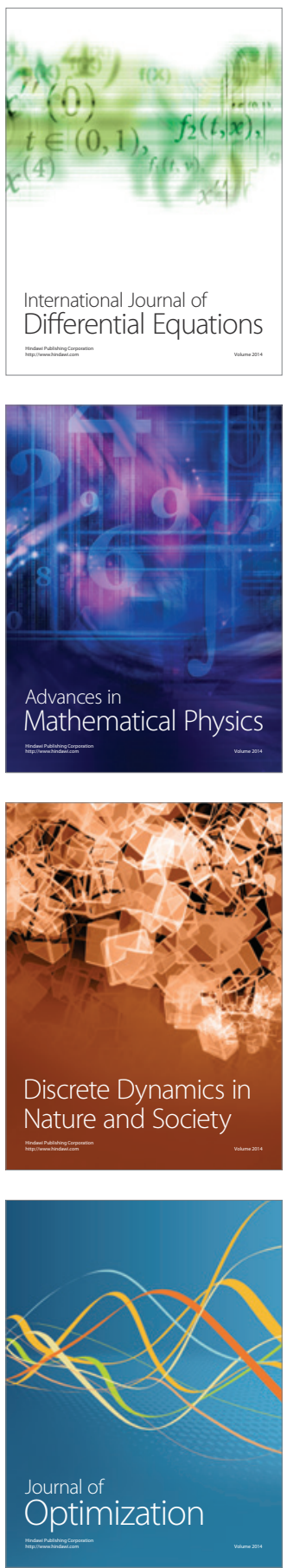